\title{
Editorial: O fascínio do conhecimento do Direito Processual Penal na era do "protagonismo jurisdicional" e da comunicação científica digital
}

\author{
Editorial: The fascination of knowledge of criminal procedural law \\ in the age of "jurisdictional protagonism" and digital scientific \\ communication
}

\section{Bruna Capparelli}

Doutoranda na Alma Mater Studiorum - Università di Bologna/Itália Editora-assistente da RBDPP bruna.capparelli2@unibo.it http://orcid.org/0000-0003-1249-2658

A Revista Brasileira de Direito Processual Penal é um periódico eletrônico que mantém as características de seletividade e de filtro dos tradicionais periódicos impressos, ${ }^{1}$ mas que aproveita a potencialidade da rede para facilitar os contatos entre os colaboradores e a transmissão dos artigos.

Pré-requisito principal de sua produção cientifica ${ }^{2}$ é, sem dúvidas, a tempestividade, ou seja, a sua capacidade de ser distribuída o mais rapidamente possível, de modo a evitar o envelhecimento ou a perda de seu valor epistemológico, por meio de mecanismos de comunicação eficazes, ${ }^{3}$ consi-

1 ALPIRANDI, Simone. Creative commons: manuale operativo. Guida all'uso delle licenze e degli altri strumenti cc. Viterbo: Nuovi equilibri, 2008, p. 11 s.

2 GOODY, Jack. Il suono e i segni. L'interfaccia tra scrittura e oralità. Milano: il Saggiatore, 1989, p. 7 s.; GOODY, Jack. La logica della scrittura e l'organizzazione della società. Torino: Einaudi, 1981, p. 56 s.; GOVONI, Paola. Un pubblico per la scienza. La divulgazione scientifica dell'Italia in formazione. Roma: Carocci, 2002, p. 43 s.; LONGO, Brunella. La nuova editoria. Mercato, strumento e linguaggi del libro in internet, Milano: Editrice Bibliografica, 2001, p. 77 s.

3 BERNARDELLI, Andrea; PELLERY, Roberto. Il parlato e lo scritto. Milano: Bompiani, 1999 e BERNARDINI, Sandro. La logica della conoscenza scientifica. Napoli: Liguori, 1980, p. 23 s. 
derado que, com o aumento da produção editorial, pareceu logo evidente que os instrumentos tradicionais de difusão dos conhecimentos - livros e revistas - não são capazes de satisfazer tal exigência com a mesma eficácia em relação ao passado.

Adota-se como meta a comunicação ao autor da decisão preliminar em até 60 dias (mas, no primeiro número de 2017, a média ficou em 30-45 dias), para posterior(es) rodada(s) de correção(ões) e publicação em até 4-6 meses (embora no primeiro número de 2017 a média da submissão até a publicação tenha ficado em 80-90 dias). Tais prazos, certamente, são mais curtos em comparação com a média das revistas nacionais de excelência, que, por experiência própria como autora, demoram de 6 a 9 meses para a decisão preliminar e de 12 a 18 meses para a publicação.

De fato, a difusão da Internet e a introdução de novos meios digitais de informação, como os e-journal, estão redimensionando a posição ocupada precedentemente pelas revistas de papel. Ademais, a RBDPP é também um fórum para a introdução e a apresentação de novas pesquisas, assim como para a crítica de pesquisas existentes: seu conteúdo assume a forma típica do artigo, contendo pesquisas originais, resenhas, etc. ${ }^{4}$

Disponibilizando gratuitamente um grande número de artigos e novos números da revista integralmente, juntamente ao extraordinário poder dos motores de pesquisa, ${ }^{5}$ tal acessibilidade torna progressivamente mais ágil a comunicação cientifica, por meio da qual os estudiosos produzem, compartilham, avaliam e difundem os resultados do próprio trabalho. De fato, trata-se de um processo que vive e se desenvolve por uma pluralidade de fatores intrinsecamente integrados; um mecanismo que, com toda evidência, encontra mudanças cuja apresentação

4 BLOOM, Harold. La saggezza dei libri. Milano: Rizzoli, 2004; BLOOM, Harold. Come si legge un libro (e perché). Milano: Rizzoli, 2000, p. 67 s.; BOLTER, Jay. David. Lo spazio dello scrivere. Computer, ipertesti e storia della scrittura. Milano: Vita e Pensiero, 1993, p. 55 s. e BOMPIANI, Valentino. Il mestiere dell'editore. Milano: Longanesi, 1998, p. 33 s.

5 BERGER, Peter; LUCKMANN, Thomas. La realtà come costruzione sociale, Bologna: il Mulino, 1969, p. 65 s. 
é muito vasta no momento em que os resultados das investigações são veiculados por suportes digitais ${ }^{6}$ e pelas redes telemáticas.

Todavia, a invasão da tecnologia na já difícil relação entre Direito Processual Penal e linguagem incrementa exponencialmente a complexa atividade comunicativa do jurista. ${ }^{7}$ A presente análise visa, portanto, a uma primeira recognição em relação aos problemas que a transmissão do conhecimento do Processo Penal, em suas várias articulações, encontra hoje. Questiona-se, em primeiro lugar, o que se entende por comunicação científica diante as novas formas de comunicação eletrônica ${ }^{8} \mathrm{e}$ as novas linguagens assumidas pelo Direito Processual Penal. Em segundo lugar, qual vínculo existe entre este meio de comunicação e a academia. ${ }^{9}$ Em terceiro lugar, quais são os circuitos intercorrentes en-

6 BAZLEN, Roberto. Scritti. In: BAZLEN, Roberto (org.). Lettere editoriali, Milano: Adelphi, 1984, p. 78 s.

7 “Os nexos e os 'ambientes circunstantes', sobretudo o conjunto. Foi e quer continuar sendo isto, o critério: até mesmo quando os caminhos se penetram e empurram a fortes distâncias. As páginas da introdução alguma coisa antecipam. Ora porém - com uma ordem complexiva circular ou talvez até invertida - é o caso retornar e escutar vozes específicas sobre aquela exigência de vínculos, aqui muito sentida. Em nome desta, seguem depois dois grandes temas: seja o uso da palavra; seja o ensinamento-aprendizado nas matérias da justiça” (NOBILI, Massimo. Giustizia, parole, insegnamento. In: NOBILI; Massimo. L'immoralità necessaria. Citazioni e percorsi nel mondo della giustizia. Bologna: il Mulino, 2009, p. 295) (tradução livre). Ver também: NOBILI, Massimo. Il processo e il diritto. In: FOFFANI, Luigi; ORLANDI, Renzo (org.). Diritto e processo penale fra separazione accademica e dialettica applicata. Bologna: BUP, 2016, p. 57 s. e CAPRIOLI, Francesco. Spunto introduttivo. In: FOFFANI, Luigi; ORLANDI, Renzo (org.). Diritto e processo penale fra separazione accademica e dialettica applicata. Bologna: BUP, 2016, p. $99 \mathrm{~s}$.

8 CAVALLARO, Renato (org.). Lexikòn. Lessico per l'analisi qualitativa nella ricerca sociale, Roma: Edizioni Cierre, 2006; DE ROBBIO, Antonella. Archivi aperti e comunicazione scientifica, Napoli: Clio-Press, 2007, p. 87 s.

9 AZZARITI, Gaetano; BURGIO, Alberto; LUCARELLI, Alberto; MASTROPAOLO, Alfio (org.). Manifesto per l'Università pubblica. Roma: Derive Approdi, 2008, p. 4 s.; BERNARDINI, Carlo. Il cervello del Paese. Che cos'è o dovrebbe essere l'Università, Roma: Mondadori Università, 2008, p. 55 s.; CAPANO, Giliberto. La politica universitaria, Bologna: il Mulino, 2000, p. 33 s.; CAPANO, Giliberto. La professione accademica. Reclutamento, carriera e retribuzione in una università autonoma e responsabile. In: TOGON, Giuseppe (org.), Una dote per il merito. Idee per la ricerca e l'università italiane, Bologna: il Mulino, 2006, p. 17 s.; EDUFACTORY 
tre dita comunicação e a sua relativa difusão na comunidade científica de referência. Em quarto lugar, qual a função ocupada pelo jurista processual-penalista e pela sua hermenêutica na sociedade da informação e da tecnologia informática. Refletir sobre o tema hoje é sempre mais urgente, diante de um quadro sistemático sobre o qual recaem grandes expectativas por parte da sociedade, mas também muitas desconfianças, $\mathrm{e}$, às vezes, abertas hostilidades.

O Direito Processual Penal tornou-se um terreno de choque cultural e institucional. E nessa colisão das polêmicas, a RBDPP almeja conquistar espaço para a reflexão: argumentada e ponderada, mas sem esconder os problemas relevantes. De fato, na era como a nossa do uso emotivo do poder e do paternalismo judiciário, o real significado da norma não emerge quase nunca do dado textual, mas frequentemente este é constituído pela síntese do confronto entre poder legislativo, judiciário e academia. Dessa forma, o uso da palavra e da linguagem consolida sempre maior importância.

De fato, é útil sublinhar como a ciência iniciou-se "com a formação de uma linguagem cientifica, isto é, com aquela linguagem que faz de um conhecimento puramente subjetivo (a minha percepção) um conhecimento ao máximo grau intersubjetivo". ${ }^{10}$ A exigência de uma linguagem científica rigorosa é relativa até mesmo às Ciências Jurídicas, especialmente em periódicos online, enquanto desafio científico, que não se identifica com os conteúdos específicos da Ciência Jurídica como conhecimento de um objeto (o ordenamento jurídico) normalmente expressado em proposições linguísticas. E na difusão do saber acessível livremente na rede telemática, a reflexão sobre as teorias gerais marcam a necessidade de uma linguagem que permita falar sobre finalidades, funções, interesses subintendidos às normas, e de organizar sistematicamente materiais normativos em relação a núcleos funcionais.

(org.). Università globale. Il nuovo mercato del sapere. Roma: Manifesto libri, 2008, p. 43 s.; ELIAS, Norbert. Coinvolgimento e distacco. Saggi di sociologia della conoscenza. Bologna: il Mulino, 1988, p. 23 s.

10 BOBBIO, Noberto. Scienza del diritto e analisi del linguaggio. In: SCARPELLI, Uberto (org.). Diritto e analisi del linguaggio. Milano: Edizioni di comunità, 1976. p. 34 s. (tradução livre). 
Feitas essas premissas, considerada a progressiva influência da comunicação digital e a consequente marginalização da editoração tradicional de Direito Processual, juristas e e-journal representam, portanto, um insubstituível vetor de determinabilidade semântica em grau de contribuir a tonar razoavelmente acessíveis e compartilháveis as reflexões científicas. ${ }^{11}$ Por outro lado, seria absurdo opor-se a estes instrumentos editoriais ${ }^{12}$ capazes de fornecer uma preciosa contribuição para a reconstrução do saber e potencialmente menos falíveis em relação às publicações impressas. Diante dessas sólidas evidências, como documenta o próprio progresso tecnológico, ganhem mais força no debate científico ulteriores aspectos filosófico-políticos e filosófico-jurídicos, capazes de interromper um curto circuito que liquidaria a possibilidade de contra argumentações: êxito perturbador visto que se considera que a ciência é o lugar da palavra e do conflito entre opostas razões, e, sobretudo, pensa-se que de tudo isso o Direito Processual Penal é decorrência direta.

Nesse quadro delineado de mudanças editoriais e de informações voláteis, ${ }^{13}$ a RBDPP oferece estudos caracterizados por rigor metodológico e sistemático, no qual se insere também os perfis problemáticos em relação ao tema da linguagem. ${ }^{14}$ Em particular, refere-se

11 EPSTEIN, Jason. Il futuro di un mestiere. Libri reali e libri virtuali, Milano: Sylvestre Bonnard, 2001, p. 47 s.; FERRAROTTI, Franco. Leggere, leggersi, Roma: Donzelli, 1998, p. 34 s.

12 UFFICIO studi dell'associazione italiana editori (org.). Investire per crescere. Materiali per una discussione, Milano: AIE, 2006, p. $54 \mathrm{~s}$.

13 MARCHETTI TRICAMO, Giuseppe; MALORINO, Tarquinio. La fabbrica delle emozioni, Come si fa l'editore in Italia, Milano: Franco Angeli, 2005, p. 45 s.; MASI, Mauro (org.). L'autore nella rete. Creatività e proprietà intellettuale nell'editoria multimediale, Milano: Guerini e associati, 2000, p. 34 s.; MCLUHAN, Marshall. Gli strumenti del comunicare, Milano: il Saggiatore, 1997, p. 39 s.; MIRANDOLA, Matilde; ATTANASIO, Pierfrancesco (org.). La nuova economia del libro. L'editoria elettronica e le professioni del libro, Milano: Istituto Poligrafico dello Stato, 1998, p. 67 s.; MONDADORI, Alberto. Lettere di una vita 1922-1975, Milano: Mondadori, 1996, p. $11 \mathrm{~s}$.

14 "Sinto a necessidade de defender alguns valores", porque agora "me parece que a linguagem seja [...] usada em modo aproximativo, casual, descuidado, e sinto um incômodo intolerável". É como "uma epidemia pestilencial" (CALVINO, Italo. Lezioni americane. Milano: Mondadori, 1993, p. 67) (tradução livre). 
à ideia geral de "lei" como símbolo de racionalidade, escudo contra o arbítrio do poder e garantia de tratamento igualitário em relação às decisões tomadas caso por caso com base em mutáveis avaliações particularistas sujeitas às influências das paixões. ${ }^{15}$ De fato, na justiça penal a palavra é a forma que contém a substância: assim como a música requer as notas encaixadas em pontuação, o Direito, e, em particular, o Direito Processual Penal, requer a palavra sintetizada em lei e em textos normativos. Que seja do Direito ou da música, a mensagem "é colocada na garrafa e lançada em mar aberto"; ${ }^{16}$ "bonito ou feio, este é entregue à escritura e o autor é plenamente responsável". ${ }^{17}$ Sendo assim, a forma parece ser o medium necessário a várias finalidades: in primis a compreensão.

O autor, jurista ou compositor, sabe que o seu texto será compreendido "segundo uma complexa estratégia de interpretação": ${ }^{18}$ essa "misteriosa e fascinante passagem" nunca é imediata, mas sempre veiculada por uma subjetiva atividade hermenêutica que pode assumir características muito diferentes, embora permaneçam imprescindíveis, de qualquer forma. ${ }^{19}$ Porém o percurso argumentativo do jurista, assim

15 Porém, como é notório, o ideal do "governo das leis", que constitui sem dúvidas uma das ideias-força da cultura ocidental, foi-se historicamente declinando em modo diferente à segunda das diversas tradições político-constitucionais. Nesse contexto, o Estado de direito é concebido como Estado legislativo, no qual o parlamento foi elevado ao centro do sistema político institucional, e o direito considerou-se inteiramente absorvido na legislação. À lei geral e abstrata emanada pelo poder legislativo foi atribuída a função de governar e proteger as liberdades, enquanto o poder judiciário teria que ter assumido uma posição subalterna, ou seja, uma posição de simples órgão aplicador do ditame legislativo, com consequente interdição do poder criativo em sede hermenêutica.

16 PULITANÒ, Domenico. Diritto penale, Torino: Giappichelli, 2009, p. 155 (tradução livre).

17 KÀROLYI, Ottó. La grammatica della musica. La teoria, le forme e gli strumenti musicali, Torino: Einaudi, 2000, p. 21 (tradução livre).

18 ECO, Umberto. I limiti dell'interpretazione. Milano: Bompiani, 1990, p. 107 (tradução livre).

19 PALAZZO, Francesco. Testo, contesto e sistema nell'interpretazione penalistica. In: VOGLIOTTI, Massimo (org.). Il tramonto della modernità giuridica. Un percorso interdisciplinare, Torino: Giappichelli, 2008, p. 252 (tradução livre). 
como o compasso na música, "desconta os limites inerentes a qualquer comunicação mediante linguagem", ${ }^{20}$ que não é nada a não ser "a essência da perfeição: contém todas as interpretações possíveis e, portanto, não limita a interpretação do intérprete”, ${ }^{21}$ porque o principal medium do direito vive de ambiguidade: combustível essencial de um processo evolutivo, sintoma claro de vitalidade do ordenamento jurídico e garantia de desenvolvimento.

Exatamente como um compositor, o jurista traduz o seu espírito criativo em sinais, dando a estes a força de sua própria autoridade. Todavia, "os sinais sobre o pentagrama são não mais do que uma ocasião": ${ }^{22}$ uma imprescindível âncora a ser conjugada em base ao contexto em que o intérprete se contorce. E se o mundo das interpretações fosse governado por uma rígida uniformidade, ${ }^{23} \mathrm{o}$ ordenamento jurídico seguiria certamente um percurso lento, completamente inadequado à mutabilidade social típica do mundo contemporâneo. Contudo, que tal mutabilidade possa ser seguida somente por meio da mutabilidade da lei, muitos consideram uma ilusão: ${ }^{24}$ esse mecanismo, às vezes perverso, ao contrário, representa o coração pulsante de todo o sistema jurídico vigente ${ }^{25}$ e, consciente desse intrigante jogo de contrapesos, com frequência é exatamente aquele legislador-compositor a limitar com precisão absoluta os confins da norma, deixando ao incessante trabalho da jurisprudência, coadjuvada pela doutrina, a especificação dos vários tipos de interpretação. ${ }^{26}$ Dessa forma o sistema jurídico ad-

20 CIONE, Edmondo. Problemi di estetica musicale, Logos, 1938, p. 25 (tradução livre).

21 PULITANÒ, Domenico. Op. cit., p. 155 (tradução livre).

22 CIONE, Edmondo. Op. cit., p. 25 (tradução livre).

23 Sobre as "regras" da complexa atividade hermenêutica, abundantemente: DAMER, Hans Georg. Verità e metodo. Milano: Bompiani, 1983, p. 11 s.

24 Assim também: HASSEMER, Winfried. Diritto giusto attraverso un linguaggio corretto? Sul divieto di analogia nel diritto penale. Ars interpretandi, 1997, p. 176.

25 MARCHESELLI, Alberto. Magistrati dietro le sbarre. Farsa e tragedia nella giustizia penale italiana, Milano: Melampo, 2009, p. 69 (tradução livre).

26 Parafraseando Sócrates em Cratilo, Gustavo Zagrebelsky recorda uma fascinante discussão sobre a interpretação na qual o filósofo adverte de "não abusar das palavras, quase como que estas sejam somente o produto de nossas arbitrárias convenções” (ZAGREBELSKY, Gustavo. Le note tra le leggi. In: 
quire flexibilidade. Os dogmas e os princípios se tornam limites da extensão, cuja função é impedir rompimentos sistemáticos com consequências nefastas.

Diante de tal panorama, discursos não menos complexos devem ser exigidos também para a comunicação cientifica digital. ${ }^{27} \mathrm{Em}$ particular, certamente deve-se ressaltar a necessidade de rigor no uso da linguagem nos textos informáticos, porque estes são iniludíveis, nos assaltam e nós os perseguimos. Às vezes desaparecem. Até mesmo nas áreas do Direito, como dito acima, esses espaços se expandem. Tendem a esconder a medida da qualidade, as proporções e as proveniências, porque, como é notório, a rede é um painel que pode conter qualquer dado, contribuindo a gerar relativismo, confusão, desorientação. Portanto, torna-se muito mais fundamental um controle rígido, com precisão linguística, porque aqueles que saberão desenvolver os próprios raciocínios com o máximo rigor e organizar de modo coerente seus pensamentos para torná-los claros e inteligíveis, se destacarão sempre como os mais persuasivos pela certeza e evidência de seu raciocínio ${ }^{28} \mathrm{E}$ isso assegura força à questão e ao seu valor, lançando um ar-

BRUNELLO, Mario; ZAGREBELSKY, Gustavo (org.). Interpretare. Dialogo tra un musicista e un giurista. Bologna: il Mulino, 2016, p. 38) (tradução livre). CADIOLI, Alberto. Letterati editori, Milano: il Saggiatore, 1995; BOURDIEU, Pierre. Gli usi sociali della scienza. Per una clinica del campo scientifico. Roma: Seam, 1999; BUCCHI, Massimiano. La scienza in pubblico. Percorsi nella comunicazione, Milano: McGraw-Hill, 2000, p. 12.

Ver: WEBER, Max. La scienza come professione. In: WEBER, Max. Il lavoro intellettuale come professione. Torino: Einaudi, 1983, p. 16 s., segundo o qual "no campo científico tem uma própria 'personalidade' somente quem serve puramente seu objeto. E isso não se verifica somente no campo científico. Não se conhece algum grande artista que não seja inteiramente dedicado ao próprio objeto e que tenha servido outros fora deste [...]. Certamente não é uma 'personalidade' aquele que, como um empresário, leva si mesmo em destaque junto com o objeto ao qual deve-se dedicar, e gostaria de justificar a si mesmo com o 'viver a própria experiência', e pergunta: como demonstro ser algo mais de um simples 'especialista', como consigo dizer algo que não tenha sido já dito por ninguém na mesma forma ou com o mesmo conteúdo? Um fenômeno, este, que hoje se observa em larga escala e deixa em qualquer lugar uma marca de mesquinhes, desanimando aquele que faz tal questionamento, onde somente a dedicação à própria tarefa pode eleva-lo à altura e à dignidade do objeto que ele pretende servir. Nem diferentemente se verifica ao artista" (tradução livre). 
gumento de discussão e atribuindo vida a seu conteúdo; caso contrário, o pensamento iria à falência e permaneceria mais uma ocasião perdida em relação à nossa responsabilidade com a transmissão do conhecimento $^{29}$ científico do Direito Processual.

\section{REFERÊNCIAS}

ALPIRANDI, Simone. Creative commons: manuale operativo. Guida all'uso delle licenze e degli altri strumenti cc. Viterbo: Nuovi equilibri, 2008.

AZZARITI, Gaetano; BURGIO, Alberto; LUCARELLI, Alberto; MASTROPAOLO, Alfio (org.). Manifesto per l'Università pubblica. Roma: Derive Approdi, 2008.

BAZLEN, Roberto. Scritti. In: BAZLEN, Roberto (org.). Lettere editoriali. Milano: Adelphi, 1984.

BERGER, Peter; LUCKMANN, Thomas. La realtà come costruzione sociale. Bologna: il Mulino, 1969.

BERNARDELLI, Andrea; PELLERY, Roberto. Il parlato e lo scritto. Milano: Bompiani, 1999.

BERNARDINI, Sandro. La logica della conoscenza scientifica. Napoli: Liguori, 1980.

BERNARDINI, Carlo. Il cervello del Paese. Che cos'è o dovrebbe essere l'Università. Roma: Mondadori Università, 2008.

BLOOM, Harold. La saggezza dei libri. Milano: Rizzoli, 2004.

BLOOM, Harold. Come si legge un libro (e perché). Milano: Rizzoli, 2000.

29 Sobre a ideia de que a Universidade não incentive a reflexão sobre si mesma, sobre a qual os acadêmicos, que por profissão e, às vezes, por vocação, ensinam a problematizar a realidade envolvente, de fato, sejam os primeiros a conferir à academia uma áurea de sacralidade, impedindo qualquer desafio em relação à mudança, ver: MORCELLINI, Mario (org.). Contro il declino dell'Università. Appunti e idee per una comunità che cambia, Milano: il Sole 24 Ore, 2005; MORIN, Edgar. I sette saperi necessari all'educazione del futuro, Milano: Raffaello Cortina, 2001, p. 43 s. e MOSCATI, Roberto. Chi governa l'università? Il mondo accademico italiano tra conversazione e mutamento. Napoli: Liguori, 1997, p. 77, os quais defendem a necessidade de uma mais acurada e profunda reflexão sobre a academia, por meio da relação entre pesquisa e ensino, vida e saber, capaz de responder às exigências de uma sociedade que está continuamente em evolução. 
BOLTER, Jay. David. Lo spazio dello scrivere. Computer, ipertesti e storia della scrittura. Milano: Vita e Pensiero, 1993.

BOMPIANI, Valentino. Il mestiere dell'editore. Milano: Longanesi, 1998.

BOURDIEU, Pierre. Gli usi sociali della scienza. Per una clinica del campo scientifico. Roma: Seam, 1999.

BUCCHI, Massimiano. La scienza in pubblico. Percorsi nella comunicazione. Milano: McGraw-Hill, 2000.

CADIOLI, Alberto. Letterati editori. Milano: il Saggiatore, 1995.

CALVINO, Italo. Lezioni americane. Milano: Mondadori, 1993.

CAPANO, Giliberto. La politica universitaria. Bologna: il Mulino, 2000.

CAPANO, Giliberto. La professione accademica. Reclutamento, carriera e retribuzione in una università autonoma e responsabile. In: TOGON, Giuseppe (org.). Una dote per il merito. Idee per la ricerca e l'università italiane. Bologna: il Mulino, 2006.

CAPRIOLI, Francesco. Spunto introduttivo. In: FOFFANI, Luigi; ORLANDI, Renzo (org.). Diritto e processo penale fra separazione accademica e dialettica applicata. Bologna: BUP, 2016, p. 99 s.

CAVALLARO, Renato (org.). Lexikòn. Lessico per l'analisi qualitativa nella ricerca sociale. Roma: Edizioni Cierre, 2006.

CIONE, Edmondo. Problemi di estetica musicale, Logos, 1938, p. 25.

DE ROBBIO, Antonella. Archivi aperti e comunicazione scientifica. Napoli: ClioPress, 2007.

ECO, Umberto. I limiti dell'interpretazione. Milano: Bompiani, 1990.

EDUFACTORY (org.). Università globale. Il nuovo mercato del sapere. Roma: Manifesto libri, 2008.

ELIAS, Norbert. Coinvolgimento e distacco. Saggi di sociologia della conoscenza. Bologna: il Mulino, 1988.

EPSTEIN, Jason. Il futuro di un mestiere. Libri reali e libri virtuali. Milano: Sylvestre Bonnard, 2001.

FERRAROTTI, Franco. Leggere, leggersi. Roma: Donzelli, 1998.

GADAMER, Hans Georg. Verità e metodo. Milano: Bompiani, 1983.

GOODY, Jack. Il suono e $i$ segni. L'interfaccia tra scrittura e oralità. Milano: il Saggiatore, 1989. 
GOODY, Jack. La logica della scrittura e l'organizzazione della società. Torino: Einaudi, 1981.

GOVONI, Paola. Un pubblico per la scienza. La divulgazione scientifica dell'Italia in formazione. Roma: Carocci, 2002.

LONGO, Brunella. La nuova editoria. Mercato, strumento e linguaggi del libro in internet. Milano: Editrice Bibliografica, 2001.

KÀROLYI, Ottó. La grammatica della musica. La teoria, le forme e gli strumenti musicali. Torino: Einaudi, 2000, p. 21.

MARCHESELLI, Alberto. Magistrati dietro le sbarre. Farsa e tragedia nella giustizia penale italiana. Milano: Melampo, 2009.

MARCHETTI TRICAMO, Giuseppe; MALORINO, Tarquinio. La fabbrica delle emozioni, Come si fa l'editore in Italia. Milano: Franco Angeli, 2005.

MASI, Mauro (org.). L'autore nella rete. Creatività e proprietà intellettuale nell'editoria multimediale. Milano: Guerini e associati, 2000.

MCLUHAN, Marshall. Gli strumenti del comunicare, Milano: il Saggiatore, 1997.

MIRANDOLA, Matilde; ATTANASIO, Pierfrancesco (org.). La nuova economia del libro. L'editoria elettronica e le professioni del libro. Milano: Istituto Poligrafico dello Stato, 1998.

MONDADORI, Alberto. Lettere di una vita 1922-1975. Milano: Mondadori, 1996.

MORCELLINI, Mario (org.). Contro il declino dell'Università. Appunti e idee per una comunità che cambia. Milano: il Sole 24 Ore, 2005.

MORIN, Edgar. I sette saperi necessari all'educazione del futuro. Milano: Raffaello Cortina, 2001.

MOSCATI, Roberto. Chi governa l'università? Il mondo accademico italiano tra conversazione e mutamento. Napoli: Liguori, 1997.

NOBILI, Massimo. Il processo e il diritto. In: FOFFANI, Luigi; ORLANDI, Renzo (org.). Diritto e processo penale fra separazione accademica e dialettica applicata. Bologna: BUP, 2016, p. 57 s.

NOBILI, Massimo. Giustizia, parole, insegnamento. In: NOBILI; Massimo. L'immoralità necessaria. Ctazioni e percorsi nel mondo della giustizia. Bologna: il Mulino, 2009. p. 295.

PALAZZO, Francesco. Testo, contesto e sistema nell'interpretazione penalistica. In: VOGLIOTTI, Massimo (org.). Il tramonto della modernità giuridica. Un percorso interdisciplinare. Torino: Giappichelli, 2008, p. 252. 
PROUST, Marcel. Il piacere della lettura. Milano: Net, 2002 (edizione fuori commercio).

PULITANÒ, Domenico. Diritto penale. Torino: Giappichelli, 2009, p. 155.

UFFICIO studi dell'associazione italiana editori (org.). Investire per crescere. Materiali per una discussione. Milano: AIE, 2006.

ZAGREBELSKY, Gustavo. Le note tra le leggi. In: BRUNELLO, Mario; ZAGREBELSKY, Gustavo (org.), Interpretare. Dialogo tra un musicista e un giurista. Bologna: il Mulino, 2016, 38 s.

WEBER, Max. La scienza come professione. In: WEBER, Max. Il lavoro intellettuale come professione. Torino: Einaudi, 1983, p. 16 s.

\section{COMO CITAR ESTE EDITORIAL:}

CAPPARELLI, Bruna. Editorial: O fascínio do conhecimento do Direito Processual Penal na era do "protagonismo jurisdicional" e da comunicação científica digital. Revista Brasileira de Direito Processual Penal, Porto Alegre, vol. 3, n. 2, p. 459-470, mai./ago. 2017. https://doi.org/10.22197/rbdpp.v3i2.79

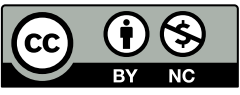

Esta obra está licenciada com uma Licença Creative Commons Atribuição-NãoComercial 4.0 Internacional. 